\title{
Study on Valve Management of DEH for Steam Turbine*
}

\author{
Changjie Yin, Jizhen Liu \\ The State Key Lab of Alternate Electrical Power System with Renewable Energy Sources, \\ North China Electric Power University, Beijing, China \\ Email: changjieyin@gmail.com
}

Received March, 2013

\begin{abstract}
Valve management is one of the major functions of DEH for steam turbine. It has an important practical significance for the security and economy of the steam turbine. This paper starts from the valve configuration figure of the domestic-type 300 MW steam turbine, and then makes a simple comparison between the two types of valve governing modes. In order to realize the valve control, the structure of control system has been established, in which the roles of the mathematical functions are discussed. On the basis of the experiment of valve flow characteristic, this article carries out a quantitative study on the functions of the valve management and the parameter tuning method. Through a serious corrections, the sequence valve flow characteristic curve is obtained, which can provide significant guidance on the research of valve management of the similar steam turbines.
\end{abstract}

Keywords: Valve Management; Steam Turbine; Digital Electro-Hydraulic Control System; Valve Flow Characteristic

\section{Introduction}

As one of the three main units in thermal power plant, steam turbine, whose rotor is in High-speed rotation under high temperature and high pressure steam, completes the conversion of heat energy to mechanical energy, while dragging the generator so that the mechanical energy into electricity. Electric power system raises two basic requirements on steam turbines used for power generation of electricity: one is guaranteed to meet the electricity needs of users at any time; the other is to enable the rotor to maintain in a certain speed, to ensure the stability of frequency of power grids and the safety of the steam turbine itself [1].

For variable load of constant pressure operation unit, the rotor speed and power regulation are achieved by changing the flow of steam. Steam volume changes can be adjusted by changing the number of opened valves and controlling the inlet area of the valves [2]. There are two types of governing modes in steam turbine, single valve (throttle governing) mode and sequential valve (nozzle governing) mode. When the steam turbine operates in single valve mode, the regulation performance of steam turbine is much better, but the throttle loss is serious; when in sequential valve mode, the valves can be adjusted to reduce the throttle loss, to improve the efficiency of the steam turbine, but the flexibility of reg-

\footnotetext{
*This work was supported by the National Basic Research Program of China ("973" Program) (Grant No. 2012CB215203) and the National Natural Science Foundation of China (Grant No. 51036002).
}

ulation performance is reduced. The appropriate valve controlling mode can improve the control quality and regulation performance of the steam turbine, thereby cutting down on coal consumption needed for power generation [3].

The Digital Electro-Hydraulic Control System (DEH), which is the best system for valve management, has been installed in almost all 300 600 MW steam turbines of Thermal Power Plants in China. DEH can switch between the two types of governing modes, whose essence is to achieve undistributed shift between throttle regulation and nozzle regulation, thus making steam turbine to achieve its best running state. This article analyzed the valve management of DEH control system of the domestic-type $300 \mathrm{MW}$ steam turbine, describing the function, principle and parameter tuning method of valve control.

\section{The Overview of Valve Control}

\subsection{The Valve Configuration Structure}

For one example generation unit, the domestic-type 300 MW steam turbine is a subcritical two-cylinder, two exhausts reheat condensing steam turbine manufactured by Dongfang Steam Turbine Company Limited. The nozzle group layout of control stage of N300-16.7/ $537 / 537$ steam turbine is shown in Figure 1. It has two high pressure main stop valves (MSV) and four high pressure main steam regulating valves (GV). The nozzle number of four nozzle groups is different, GV1 valve controls the number of nozzles 32; GV2 valve controls 
the number of nozzles 32; GV3 valve controls the number of nozzles 30; GV4 valve controls the number of nozzles 27 . When the steam turbine is in a normal operation, the main stop valves are fully opened and the steam flow is controlled by the four control valves.

\subsection{Comparison of the Single Valve Mode and Sequence Valve Mode}

For the constant pressure operation of power generation units, steam turbine regulation is mainly to regulate the speed of rotor and the power of generator, which are achieved by regulating the steam flow. The amount of inlet flow is altered by changing the number of opened valves and valve opening, namely changing the total inlet area of steam turbine. Therefore, depending on how to change the inlet area of valves, we can divide the control mode into two types: single valve mode and sequential valve mode, which have their own advantages and disadvantages.

Single valve control means that all the control valves accept a valve control signal to make the valves turn up or down at the same time, which is characterized by the throttle adjusting and full arc admission. The cylinder rotor heat expansion is uniform and the metal temperature of different steam turbine parts is in a stable condition, making the unit withstand greater load change rate. But because all of the adjustment valves are not in the fully opened state, the valves have a great throttle loss, reducing the thermal efficiency of the unit.

Sequence valve control means that the control valve turns on or off individually along with the rotor speed or turbine load changing, which is characterized by the nozzle adjusting and partial arc admission. That is to say, at any time only one steam valve is in a non-fully open state while the other valves are in fully open state or fully closed state. This control mode reduces the throttling losses and thereby improves the thermal efficiency of the steam turbine. But because the position of the steam inlet is asymmetric, the cylinder rotor heat expansion is uneven and the metal temperature difference of different steam turbine parts is rather large, and the unit cannot withstand greater load change rate.

Figure 2 is the 300 MW unit's thermal efficiency curve of single valve control and sequence valve control. When the load percentage is $60 \%$, sequence valve mode can improve thermal efficiency by about $3 \%$.

\section{The Principle of the Valve Control}

The function of valve control is to transfer the required flow into the degree of valve opening. In order to realize the function, there are several mathematical functions used to correct the flow and distributing it among the four valves. For a valve, the valve control structure is shown in Figure 3, in which the roles of functions are explained as follows.

\subsection{Flow Pressure Correction Function $f\left(x_{1}\right)$ and $f\left(x_{4}\right)$}

Function $f\left(x_{1}\right)$ and $f\left(x_{4}\right)$ are correction functions between the unit's theoretical demand flow and the actual demand flow. Under low load condition, actual demand flow is equal to the theoretical demand flow. But as the load increases, the pressure of steam turbine governing stage rises and its actual amount of steam decreases although under the same valve opening. That is to say actual demand flow is higher than the theoretical demand flow. Therefore, the functions are used for amending the flow directive at different load levels, to ensure the consistency of the theoretical flow and the actual flow.

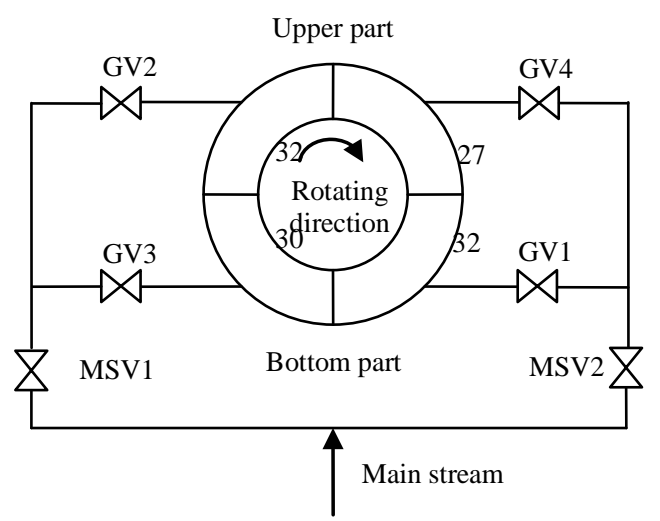

Figure 1. The nozzle groups structure chart of control stage.

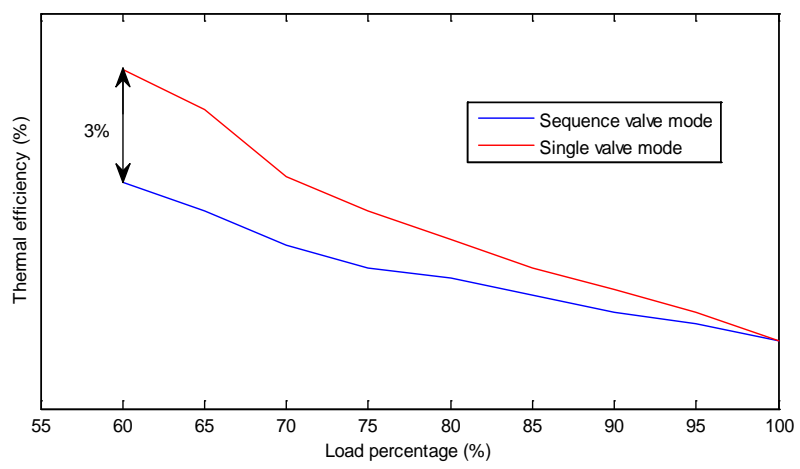

Figure 2. Thermal efficiency comparison chart.

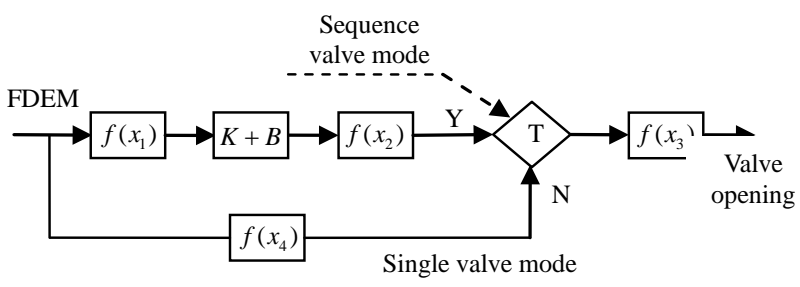

Figure 3. Functional structure of valve control system. 


\subsection{Flow Proportion and Bias Factor $K+B$}

When the steam turbine is under single valve control, all the valves are directly controlled by the flow instruction, so proportion and bias factor are not needed. When the steam turbine is under sequence valve control, the valves are opened one by one, so it need to give each valve the proportion and bias factor, to ensure that the valve is opened according to the designed order.

\subsection{Valve Overlap Correction Function $f\left(x_{2}\right)$}

In the sequence valve control mode, the valves are sequentially opened. If a valve is opened after the full opening of another, the relationship between the total valve lift and flow is squiggle according to a single valve characteristic [4]. In order to make the curve linearism, it is needed to open the next valve in advance before the previous valve being fully opened. How much the next valve is opened is controlled by $f\left(x_{2}\right)$.

\subsection{Valve Flow Characteristic Curve $f\left(x_{3}\right)$}

Valve flow characteristic curve, which convert the flow instruction into the valve opening instruction, is the oneto-one correspondence between the valve opening and steam flow through the valve. The curve is determined by the physical characteristics of the valve, such as valve lift and valve area.

\subsection{The Undisturbed Switch between Single and Sequence Valve Mode}

During unit start-up and load changing, the valve management in the control system is set at single valve control mode to ensure full arc steam admission around the nozzles to achieve uniform heating and reduce thermal stress. Under stable load operation, the system is shifted into sequence valve control mode to reduce the throttling loss caused by full arc steam admission and improve the thermal efficiency. In order not to affect the stability of the unit output, the switching process must be smoothly operated without any perturbations. One of the excellent features of DEH is the undisturbed switch between the two steam governing methods.

Firstly, valve management program calculates the final flow for each valve under the two control modes at the same time. When the mode is switched from single valve mode to sequence valve mode, the final flow of each valve calculated under single valve mode is changed to the final flow calculated under sequence valve mode in a certain rate, and as a result, the opening of each valve is approaching to the position required by sequence valve mode. The switching process is completed after all valves reaching their required opening position. During the conversion process, some valves must be opened in- creasingly, while the others closed gradually. At any moment throughout the process, increased flow and decreased flow should be equal, so that the total flow remains unchanged. Thus, the load of power unit in the conversion process will not be affected, but thermal efficiency is higher than in the sequence valve control mode, so electric power will increase after the switching. When the sequence valve control is switched to single valve control, the process is reversed though the principle is the same,

Figure 4 is the switching logic between sequence valve mode and single valve mode.

The switching logic is expressed as follows

$$
\mu=X_{1} \times \mu_{1}+X_{2} \times \mu_{2}
$$

where $\mu$ is the final degree of valve opening, $\mu_{1}$ is the degree of sequence valve opening, $\mu_{2}$ is the degree of single valve opening, $X_{1}$ is sequence valve coefficient and $X_{2}$ is single valve coefficient.

The relationship between the two coefficients is described as follows

$$
X_{1}+X_{2}=1
$$

The essence of the switching process from single valve mode to sequence valve mode is that the coefficient $X_{1}$ transforms from 0 to 1 according to a certain rate while $X_{2}$ transforms from 1 to 0 according to Equation (2). The coefficient $X_{2}$ variation with time is shown Figure 5 [5].

The switching rate is decided by the Division of logic circuits: 0.2/120.In Figure 5, the period of logic circuits is 0.2 seconds, so after 12 seconds, coefficient $X_{1}$ becomes 0.9; after 120 seconds, coefficient $X_{1}$ becomes 0 . In order to make the switching process much more stable, the denominator can be modified to 180 , and then the conversion time is extended to 180 seconds.

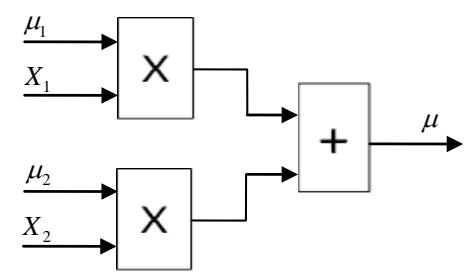

Figure 4. Switching logic.

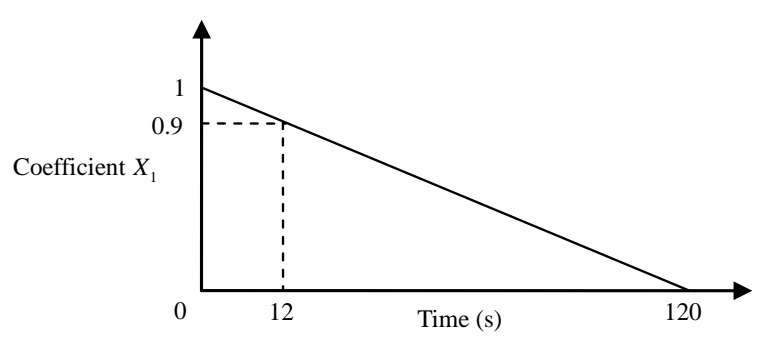

Figure 5.Coefficient $X_{2}$ variation with time. 


\section{Parameter Tuning of Valve Control}

In Figure 3, $f\left(x_{1}\right), f\left(x_{3}\right)$ and $f\left(x_{4}\right)$ are decided by the attribute of the steam turbine, so generally, they are provided by the steam turbine factory. Therefore, this article only discusses sequence valve control parameter tuning.

In this mode, the given flow value of FDEM firstly is amended by flow pressure correction function $f\left(x_{1}\right)$, flow proportion and bias factor $K+B$, valve overlap correction function $f\left(x_{2}\right)$, and then through valve flow characteristic curve $f\left(x_{3}\right)$, turned into the final valve opening instruction. The experiment of valve flow characteristic was carried out in the 300MW capacity steam turbine. Much more details about the tests can be found in [6].The experimental data after processed is as shown in Table 1.

\subsection{Flow Pressure Correction Function $f\left(x_{1}\right)$}

Function $f\left(x_{1}\right)$, provided by the turbine manufacturer, is set as shown in Table 2.

\subsection{Flow Proportion and Bias Factor $K+B$}

$K$ and $B$ are determined by the design flow rate and the opening sequence of the valve. When the steam turbine is in sequential valve operation, GV1 and GV2 are opened at the same time while GV3 and GV4 opened in sequence order under the consideration of degree of overlap.

According to the design data, when FDEM is $0 \%$ (the value is $0 \%$ after amended by function $f\left(x_{1}\right)$ ), the flow instructions of GV1 and GV2 are $0 \%$; when FDEM is $78.7 \%$ (the value is $80.3 \%$ after amended by function $f\left(x_{1}\right)$ ), the flow instructions of GV1 and GV2 are $100 \%$, that is to say, the two valves are opened fully. So by the following formula:

$$
\left\{\begin{array}{c}
0=K \times 0+B \\
100=K \times 80.3+B
\end{array}\right.
$$

One can get the result: $K_{3}=1.239, B_{3}=0$

GV3 is opened after GV1 and GV2 fully opened. So when FDEM is $76.3 \%$ (the value is $77.5 \%$ after amended by function $f\left(x_{1}\right)$ ), the flow instruction of GV3 is $0 \%$; when FDEM is $93.3 \%$ (the value is $107.1 \%$ after amended by function $f\left(x_{2}\right)$ ), the flow instruction of GV3 is $100 \%$, that is to say, GV3 is opened fully. So by the following formula:

$$
\left\{\begin{array}{c}
0=K \times 77.5+B \\
100=K \times 107.1+B
\end{array}\right.
$$

\begin{tabular}{|c|c|c|c|c|c|c|}
\hline $\begin{array}{c}\text { Flow } \\
\text { Instruction } \\
\text { (\%) }\end{array}$ & $\begin{array}{l}\text { Power } \\
\text { (MW) }\end{array}$ & $\begin{array}{l}\text { Main stream } \\
\text { pressure } \\
(\mathrm{MPa})\end{array}$ & $\begin{array}{l}\text { Pressure after } \\
\text { regulating stage } \\
(\mathrm{MPa})\end{array}$ & $\begin{array}{c}\text { The opening instruction } \\
\text { of GV1 and GV2 } \\
(\%)\end{array}$ & $\begin{array}{l}\text { The opening } \\
\text { instruction of GV3 } \\
\text { (\%) }\end{array}$ & $\begin{array}{l}\text { The opening } \\
\text { instruction of GV4 } \\
\text { (\%) }\end{array}$ \\
\hline 68.0 & 184.4 & 15.411 & 7.450 & 39.2 & 0.1 & -0.2 \\
\hline 68.2 & 188.7 & 15.354 & 7.674 & 40.3 & 0.6 & -0.2 \\
\hline 68.6 & 199.6 & 15.504 & 8.252 & 42.8 & 1.8 & -0.2 \\
\hline 69.2 & 214.8 & 15.617 & 8.851 & 45.8 & 3.3 & -0.2 \\
\hline 70.1 & 225.3 & 15.630 & 9.311 & 51.0 & 6.0 & -0.2 \\
\hline 71.1 & 232.0 & 15.379 & 9.478 & 56.5 & 8.5 & -0.2 \\
\hline 72.1 & 237.0 & 15.421 & 9.686 & 62.1 & 11.0 & -0.2 \\
\hline 73.1 & 237.8 & 15.379 & 9.762 & 67.2 & 11.4 & 0.0 \\
\hline 75.1 & 241.9 & 15.298 & 9.846 & 78.7 & 12.1 & 0.0 \\
\hline 77.1 & 244.9 & 15.454 & 9.990 & 88.7 & 12.8 & 0.0 \\
\hline 79.1 & 247.3 & 15.548 & 10.080 & 98.0 & 13.7 & 0.0 \\
\hline 81.1 & 246.4 & 15.561 & 10.084 & 98.0 & 15.6 & 0.0 \\
\hline 83.1 & 247.3 & 15.454 & 10.152 & 98.0 & 18.2 & 0.0 \\
\hline 85.1 & 250.8 & 15.292 & 10.292 & 98.0 & 20.5 & 0.0 \\
\hline 87.1 & 257.7 & 15.304 & 10.631 & 98.0 & 24.3 & 0.0 \\
\hline 88.1 & 275.3 & 15.461 & 11.319 & 98.0 & 32.6 & 9.9 \\
\hline 88.5 & 280.4 & 15.461 & 11.557 & 98.0 & 36.0 & 10.0 \\
\hline 90.5 & 283.0 & 15.398 & 11.720 & 98.0 & 56.3 & 11.0 \\
\hline 92.5 & 284.6 & 15.392 & 11.765 & 98.0 & 84.2 & 12.3 \\
\hline 95.0 & 285.8 & 15.411 & 11.844 & 98.0 & 98.0 & 16.1 \\
\hline 97.0 & 287.2 & 15.173 & 11.924 & 98.0 & 98.0 & 21.4 \\
\hline 98.0 & 288.7 & 15.066 & 12.016 & 98.0 & 98.0 & 25.9 \\
\hline 99.0 & 290.0 & 14.960 & 12.137 & 98.0 & 98.0 & 34.0 \\
\hline 100.0 & 294.2 & 14.972 & 12.332 & 98.0 & 98.0 & 98.0 \\
\hline
\end{tabular}

Table 1. Valve flow characteristic test data under sequence valve mode. 
Table 2. The functional settings of flow pressure correctiom.

\begin{tabular}{cc}
\hline Flow instruction (\%) & Flow instruction after amended (\%) \\
\hline 0 & 0 \\
60.011 & 60.011 \\
71 & 71.428 \\
74 & 74.732 \\
79 & 80.686 \\
86 & 90.928 \\
100 & 122.025 \\
\hline
\end{tabular}

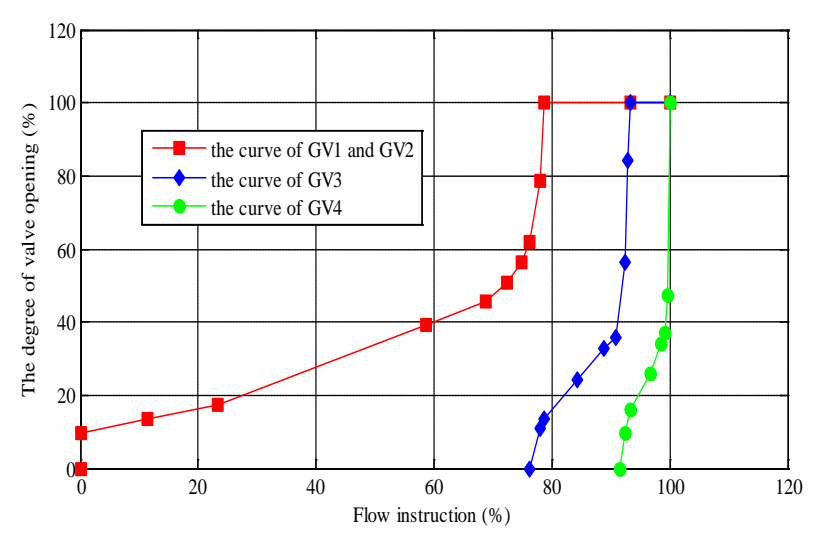

Figure 6.Valve flow characteristic curve.

One can get the result: $K_{3}=3.378, B_{3}=-261.8$.

GV4 is opened after GV1, GV2and GV3 fully opened. So when FDEM is $91.6 \%$ (the value is $103.4 \%$ after amended by function $f\left(x_{1}\right)$ ), the flow instruction of GV3 is $0 \%$; when FDEM is $100 \%$ (the value is $122 \%$ after amended by function $f\left(x_{2}\right)$ ), the flow instruction of GV3 is $100 \%$, that is to say, GV4 is opened fully. So by the following formula:

$$
\left\{\begin{array}{l}
0=K \times 103.4+B \\
100=K \times 122+B
\end{array}\right.
$$

One can get the result: $K_{4}=5.376, B_{4}=-555.9$.

\subsection{Valve Overlap Correction Function $f\left(x_{2}\right)$}

Generally speaking, when the ratio between the main stream pressure and the stream pressure in the back of the previous valve is $0.85 \sim 0.9$, the next valve starts to open. In this paper, based on observing the test data of Table 1, the degree of valve overlap is obtained by the graphing method [4].

Through the above corrections, the final valve flow characteristic curve is shown in Figure 6.

\section{Conclusions}

Valve management is an important aspect of Digital Electro-Hydraulic Control System, which is directly related to the security and economy of unit operation. This article analyzed the DEH control system of the domestic type 300 MW steam turbine, described the function, principle and parameter turn method of valve control, and finally obtained the valve flow characteristic curve in sequence valve mode. The study in this paper can pro vide significant guidance on the research of valve management of the similar steam turbine.

\section{REFERENCES}

[1] S. X. Wang and X. X. Ge, "Digital Electro-hydraulic Control System of Steam Turbine,” 1st Edition, China Electric Power Press Co., Ltd., Beijing, 2004.

[2] D. R. Yu, J. F. Liu and J. Y. Xu, et al., "The New Method Reducing Governing Valves' Throttling Loss,” Turbine Technology, Vol. 42, No. 5, 2000, pp. 271-273.

[3] W. L. Liu, P. F. Liu and X. H. Hu, et al., "Effect of Thermal Automatic Control on Energy-saving and Consumption-reducing in 600 MW Power Unit," Electric power, Vol. 43, No. 10, 2010, pp. 44-47.

[4] S. F. Tian, Z. J. Shi and L. T. Yan, "Study of Valve Overlapping of Steam Turbine Control System,” Turbine Technology, Vol. 50, No. 6, 2008, pp. 448-450.

[5] K. K. Zhou and J. J. Gu, "Given Value Processing and Valve Management System with DEH,” Electric Power Science and Engineering, No. 4, 2006, pp. 80-83.

[6] Z. Y. Wen and W. Lu, "Improvement of Steam Turbine Valve Characteristic Optimization Approach," China Electric Power Education, Vol. S1, 2010, pp. 692-693. 